\title{
A WEB-BASED CENTRAL GATEWAY INFRASTRUCTURE IN THE AUTOMOTIVE AFTER-SALES MARKET Business interoperability through the web
}

\author{
Geert Houben, Kris Luyten, Karin Coninx \\ Hasselt University - $t U L$ - Expertise Centre for Digital Media \\ Wetenschapspark, 2, BE-3590 Diepenbeek (Belgium) \\ Email: \{geert.houben,kris.luyten,karin.coninx\}@uhasselt.be \\ Frank Schönherr \\ MUL-Services $\mathrm{GmbH}$ \\ Pontdriesch, 10, D-52062 Aachen (Germany) \\ Email: f.schoenherr@mul-services.de
}

Keywords: automotive aftermarket, technical information, SOA, e-procurement, EAI, service provisioning

Abstract: The Block Excemption Regulation of the European Commission was enacted in 2002 with the goal to strengthen competition between dependent and independent repairers in the automotive after-sales market. The FP6 MYCAREVENT project embraces these goals while triggering new business opportunities by establishing a mobile accessible infrastructure as single gateway to different kinds of resources. This information procurement framework allows customers to find specific vehicle repair and diagnostic data from different car manufacturers and $3^{r d}$ parties in the same way. In order to provide a higher degree of accessibility, extensibility and adaptivity, our service-oriented infrastructure presented in this paper is web-based and consists of three main components: Mobile Clients, Service Portal and Remote Services. New communication and multimedia technologies are invoked to improve interoperability, usability and maintenance of the underlying Mobile Service World. In this paper we focus on the architecture of our highly flexible procurement infrastructure. Standardized elements and methodologies ensure an integrated solution and enable easy expandability with new content, services and components.

\section{INTRODUCTION}

The European automotive market is known as being very competitive due to the large amount of car manufacturers (OEMs). The after-sales market is just a branch of this industry but plays a very important role from a business perspective ( $€ 40$ billion p.a. volume), and will become even more important in the future. In the FP6 MYCAREVENT project, new services and innovative applications are being developed to support different types of end-users to cope with the increasing -electronic- complexity of cars and their repair procedures. New communication and multimedia technologies are exploited to improve the accessibility and usability of the information and services, which can be seamlessly and securely accessed by different types of mobile devices ((Houben et al., 2005a)). End-users in the sense of MYCAREVENT are workshop mechanics, roadside technicians, support call-centre operators and the driver.

Car manufacturers have a comprehensible interest to protect their IPR and to safeguard market shares.
But internal secrecy of vehicle related repair information could cause market restrictions for the after-sales sector. The Block Exemption Regulation (BER) of the European Commission ((FIGIEFA, 2002)) targets this issue to widely open the market to independent repairers as well as enabling further business opportunities. By giving all service providers the same right to access different kinds of repair and diagnosis information, training material and tools, the competition and competitiveness of the after-sales market will be strengthened. However, there is only limited collaboration among the key players in this field and the traditional business models and processes do not facilitate interaction among market participants. The project aims to create a win-win-situation for all key players by establishing a suitable framework to support this collaboration. We call the collection of services, users and content the MYCAREVENT Mobile Service World, see Figure 1.

The key innovation in the project is the seamless integration of novel services and different information sources via a Service Portal as single point of 


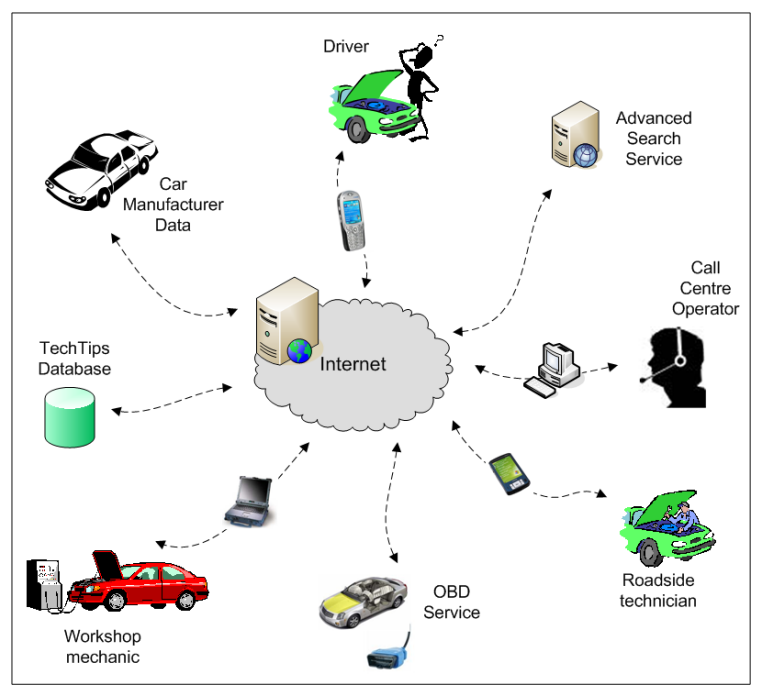

Figure 1: The MYCAREVENT Mobile Service World

access for end-users. To enable this integration and to harmonize the access of information from a huge variety of repositories, we present a unified definition of car-repair service interfaces together with a webservice oriented middleware. Companies are able to host their own services and share information regardless of the underlying software platforms. Standardized XML messaging, a generic workflow and consistent meta-data structures are required for providing advanced search and diagnosis mechanisms as well as unified user interfaces to access this information.

Mobility is an important requirement: the degree of accessibility of the Internet these days allows the customer to be connected whenever and wherever. The MYCAREVENT infrastructure is a network of several interrelated web entities: web servers that are connected over the Internet and communicating using XML web services (Booth et al., 2004). This implies considerable advantages regarding software updates, accessibility, modularity and integration of new services. Nevertheless such a loosely coupled interorganizational network requires predefined communication rules and well-defined interfaces.

A detailed project requirement analyzes phase mainly revealed four important aspects, not only relevant within MYCAREVENT:

- Flexibility in the supported business models to cover existing market situations and to increase acceptance on user and on provider side

- Integration of various and inhomogeneous kinds of information structures and repositories

- Providing a unified/homogeneous user interface and search functionality to facilitate easy access to repair information
- Expandability towards future services and (meta) data requirements which could arise e.g. from follow-up regulations of the current BER

In this paper we focus on the architecture of the information procurement infrastructure and the necessary elements that contribute to the smooth integration and cooperation of isolated services or information repositories. During the last two years of MYCAREVENT a prototype with a short time-to-market is developed and will be refined, amended and finalised in the upcoming year. Business and operator models are defined for the exploitation phase of the product, but are out of the scope of this paper ((Garg et al., 2004)). In the following section we describe in more detail the scope, the goal, the challenges, the benefits and the target user groups our architecture has to deal with.

\section{PROCUREMENT INFRASTRUCTURE}

The Block Exemption Regulation (BER) provides the legal framework for regulations regarding the distribution and maintenance of motor vehicles. MYCAREVENT offers a uniform and generic solution which not only covers the BER regulation but also allows the user to compare information and offers access to advanced services (examples are provided further in the text). We call this framework the 'repair information procurement infrastructure' as it is directed form the information supplier to the customer.

When having a closer look on the automotive aftermarket, a huge collection of technical information exists within car companies, independent roadside assistance services and driver organisations. Bringing this content together, linking it with diagnostic information and offering unified access to it via a single mobile accessible platform will allow manufacturerdependent as well as independent mechanics and also drivers ${ }^{1}$ to speed up information retrieval and consequently repair procedures. Market analysis ((MYCAREVENT, 2005)) have shown huge benefits for the different user groups. This paper will mainly focus on the architecture and technological challenges.

A major challenge is the remarkable diversity in the structuring, naming and composition of data within different car companies and other content providers. This heterogeneity requires a well-defined,

\footnotetext{
${ }^{1}$ It is not intended by MYCAREVENT to provide the driver with the same kind of repair information than professional mechanics
} 
standardized MYCAREVENT data model that is flexible, adaptable and implemented across the different content providers. The data model, developed within the project, is a meta model; it describes the data and extends it with uniform properties to make it accessible and analysable by a wide variety of advanced search mechanisms, like Ontologies ((Bryan and Wright, 2005)) or Expert systems. This approach permits leaving the original content provider/OEM data largely untouched, having in mind that forcing a change of existing data provider structures easily can incur unpredictable costs as it targets related authoring systems and processes.

The data model concept described in the previous paragraph forms the basis for the message format that is defined to enable standardized communication between the various components and services. The flow of information and the order of interaction between the end-user and the system are laid down by the generic workflow steps. Communication links are established over the standard Internet protocol: SOAP envelopes are used to circulate generic XML messages over HTTP. Identical web service interfaces are implemented on top of every component. Using the Internet for communicating offers advantages regarding mobility, adaptivity, ubiquity and extensibility. Nevertheless the use of an open media requires a secure and authenticated communication channel, e.g. via VPN-routers as a cost-efficient and wellestablished industrial standard.

The web service interfaces, the standardized messaging and the generic workflow are the three basic elements for communication between previously isolated entities. Due to these elements, it is clear and well defined respectively how to communicate, what to communicate and in which order to communicate.

Three main parts constitute the infrastructure: Mobile Clients (accessing the information), the Service Portal (core of the infrastructure) and the Remote Services (providing services or information). The Service Portal is the core component that channels the user demand through a single, comprehensive gateway. It covers flexible business rules and basic requirements, such as user management, to enable a wide variety of functionalities such as multi-language search and diagnosis, information distribution, hosting and authoring of repair information. Eventually it makes centrally accessible the range of MYCAREVENT Remote Services.

Examples of services and content in the MYCAREVENT Mobile Service World are: Interactive Circuit Diagrams to repair electronic components in the car, Technical Tips about generally operated reparations or fixes on specific car models, Electronic
Driver Manuals with animations and graphical data, Diagnostic Services to analyse vehicle fault codes, an Expert System to lead the user through the diagnosis of a car breakdown, Value Added Services for the driver (infotainment, news, traffic information, simple guided car reparations), an Advanced Query Service for searching repair information by making use of ontologies.

The MYCAREVENT infrastructure offers multiple benefits to the aftermarket. Services and content from different companies and organisations are integrated into a single service portal that is 'always, everywhere and uniformly' accessible on different mobile devices. By its interoperability between different providers, services that were previously stand-alone entities can now be combined and amended with the further information available in the infrastructure. It brings vehicle information handling, gathering, maintenance and repair to a higher level of use; for example, in the case of a breakdown, linking the fault code of the car with simple self-repair instructions (movie, pictures, etc.) ((Houben et al., 2005b)); or answering iterative hierarchical questions to an Expert System which eventually provides the most probable error and an advice whether it is safe to continuing driving or not.

On the repair level, independent workshops or roadside assistance companies are able to easily access OEM information, which allows them to perform even complex repairs. Repair and diagnosis procedures are speeded up, success rates are enhanced while sharing infrastructure costs, resulting eventually in higher customer satisfaction. On the level of information provision via the infrastructure; by providing different information from different resources, it can be easily compared, in content and price.

\section{SYSTEM ARCHITECTURE}

In this section the architecture of the repair information procurement framework is described. The basic flow of information between mobile clients, the portal and the remote services is defined as follows: the client requests information from the portal, the portal routes this request to the appropriate service(s), these service(s) respond to the portal, which forwards the response to the client. These workflow steps are elaborated in Section 3.2. The communication between the components is supported by XML-based messages and corresponding web services. Web services allow us to use the standard HTTP protocol without incurring firewall restrictions or interoperability problems. 


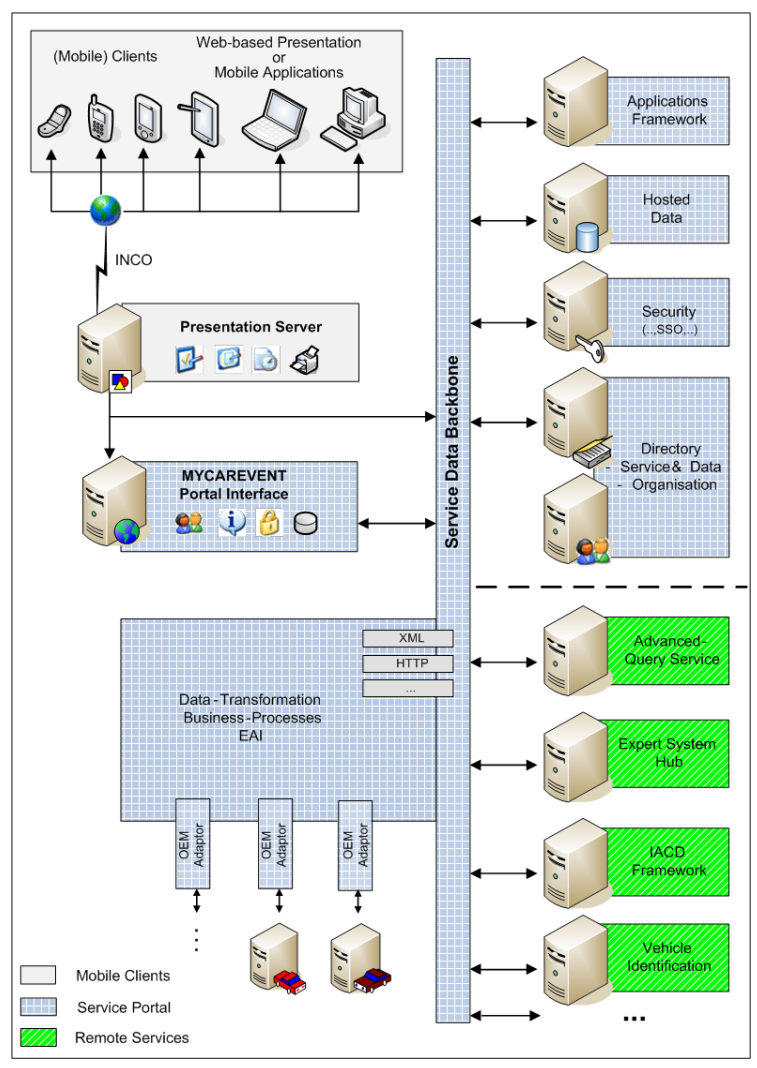

Figure 2: Architectural overview of the procurement framework

The clients of the Service Portal are the mobile application providers offering the user interface for utilizing and querying the portal. In Section 3.1 we will focus on such a client that has been implemented as part of the presented infrastructure: the Presentation Server. The Presentation Server translates the portal messages into human readable web pages and serves as the interaction layer between the end-user and the MYCAREVENT infrastructure.

\subsection{System Components}

Figure 2 shows the architectural overview of the infrastructure. This architecture enables the development of different presentation engines (user interfaces) or end-user applications, related to the business model of the respective provider. These applications can depend on the envisaged target user group, the envisaged information provisioning, the envisaged target device etc. In the prototype we have created, currently there is one direct consumer of the portal web service interface: the Presentation Server (PS).

The PS is a web server that consists of two main parts: a message engine to communicate with the Service Portal and a visualization engine that provides the user interface. The visualization engine trans- forms the portal messages into user readable and interactive web pages accessible with a standard web browser. This web-based approach has several benefits: a standard web browser can be used and is available on nearly all platforms; updates are done on the server instead of updating all the clients; processing the data at runtime is done server-side and does not rely on the client. This last advantage ensures the required capabilities for client devices to interact with the PS are limited and a wide range of devices can be used. There are also some disadvantages or restrictions using the web-based approach, e.g. limited graphical support, browser plug-ins needed for dedicated or advanced functionality, but after all it fits very well the requirements and idea of 'ubiquity' of MYCAREVENT. The client terminals in figure 2 are connected to the Service Portal/Presentation Server by using a Intelligent Connection (INCO) which provides seamless connectivity regardless of the used radio access system (GSM, UMTS, WLAN, ...), see e.g. (Gehlen et al., 2006) for more details.

The Service Portal answers the incoming PS requests with downloaded data to the client devices like repair/diagnosis information - or with routing the request to the appropriate services. Multiple Remote Services can be simultaneously queried within the same user inquiry to provide the customer supplementary and diversified results. The direct querying and comparison of different providers enables transparent user decisions. When e.g. an independent roadside assistance organisation provides technical tips on reparations for a specific car type, in similar cases these tips could be more useful than buying the official OEM repair manual for twice the price. But price comparison is not the most important benefit; the customer gets a better and more specific offer due to the extensive range of content and information that is available on the one hand and because of the delivered data is tailored to the user situation on the other hand.

In Section 2 we already gave some examples for Remote Services. The next example shows the use of it. Think of the following scenario: your car has broken down and the roadside technician accesses the MYCAREVENT system with a dedicated application on his PDA. The PDA connects with the car diagnosis plug (OBD) over Bluetooth, reads out the fault code and sends it to the portal over GPRS. The portal forwards the code to the diagnosis services, which translates it and returns a description of this fault in MYCAREVENT terms (according to the information model). The portal forwards this fault description to the Advanced Query Service, which answers with document search results related to that fault, even if 


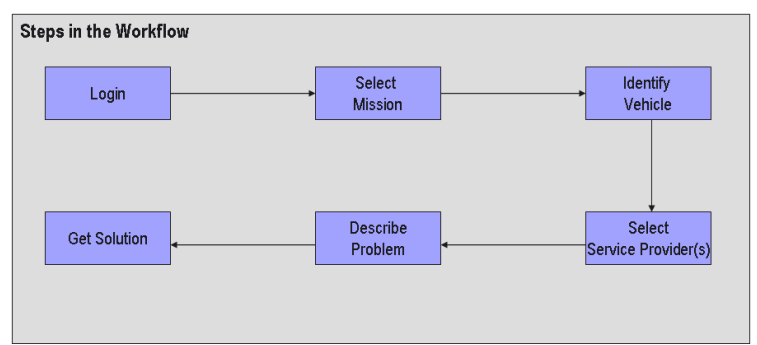

Figure 3: The main steps of the information procurement generic workflow

the fault or document itself is not expressed in MYCAREVENT terminology (see the meta model concept above). The list of solutions is sent to the technician who downloads the document(s) that are required to fix the problem.

\subsection{Generic Workflow}

Information procurement with the MYCAREVENT infrastructure is a stepwise and well-defined process, divided into six successive steps (Figure 3). This workflow defines the flow of information and the implicit and explicit user interactions with the system.

The first step in the workflow is the user authentication. Secondly the user has to choose a mission from the mission menu. Examples of missions that are included in the implemented prototype are: searching for repair information, training on using the infrastructure, vehicle diagnosis, driver self-help etc. The third step is vehicle identification. Accessing repair or diagnostic information makes in most cases only sense when the particular vehicle is identified or at least the make and model are known. Based on further car properties like sales model, year of manufacturing or fuel type, services can decide if they are able to provide useful information or request via messaging more details. Selecting or deselecting (filtering) particular services for being multi-queried is step four.

In the fifth step the queried services have the opportunity to request further information from the user in order to provide more appropriate solutions. E.g. in the 'Repair information' mission the services can ask the user via the PS for the car component and subcomponent (e.g. 'Washer system', 'Headlight washer') or a symptom description (e.g. 'Headlight washer does not function anymore'). The sixth and last step is the solution retrieval. As the infrastructure is web-based, the solution is downloaded from the portal or from a content provider.

Figure 4 illustrates the situation in the current prototype, comparable to the geographical 'widespread' of MYCAREVENT users, service and data providers.

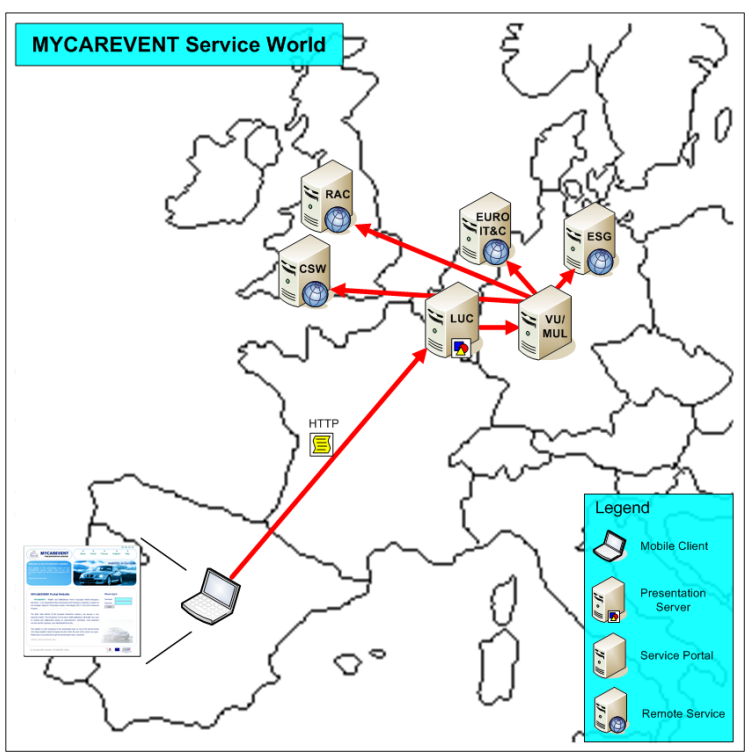

Figure 4: Flow of information in the MYCAREVENT infrastructure

For example, a client in Spain uses a laptop to access the MYCAREVENT framework over the Internet. The user communicates with the Presentation Server that translates the HTTP requests into messages conform with the standardized message structure. These messages are sent to the portal, which forwards them to the appropriate services. The portal bundles the responses from the services and sent the result to the Presentation Server that generates HTML pages which can be accessed by the client that initiated the request. This process is repeated for each step in the workflow.

\subsection{Message Definition}

The web service communication between the components is not method-based, but uses XML-based messages to interact with the web services. Only a single method is therefore required in the web service layers that takes care of the messages: MCEMessage SendMessage(MCEMessage).

In the introduction of this paper we described the unified access to different sources of information from different companies/information providers. As these sources have no uniform data structures, a standardized meta-model has to be created to embed the original information in or to 'describe' the information. This allows search engines to find the relevant information. The data model, defined in the project to deal with these issues, is called GIIRM (Generic Integrated Information Reference Model, (MYCAREVENT, 2006)). It reflects and recognizes relationships between faults, vehicle systems, symp- 
toms, repair information etc. which enables 'intelligent' searching.

The MYCAREVENT message structure is an aggregation of different parts of the data model. Classes of meta data or bundles of information (IB) are defined to describe the different parts of the GIIRM. Information model categories exist for user profile, mission description, vehicle identification, problem description, solution and so on. These parts match the generic workflow steps and are incrementally added to the messages during the procurement process. The messages are stateless; all relevant information is available at any time in the process.

Listing 1: VehicleTypeIdentification Information Bundle as part of a MCEMessage

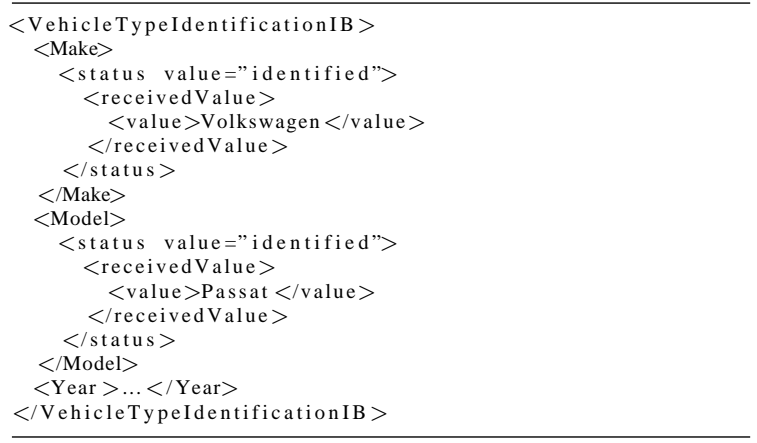

To draw an example: the user is already logged in on the portal, the next step is choosing the mission. The PS sends a message to the portal including a MissionDescription IB. The portal responds with the missions that are available for the current user profile and user role. For every other workflow step a new information bundle is added to the message or user/system selected values inside IBs are changed. After the identification of the vehicle, the VehicleTypeIdentification IB could look like the one in Listing 1.

\section{CONCLUSION}

The introduction of new mobile services and applications into the automotive aftersales market will enable new ways of collaboration and business opportunities among car manufacturers, roadside assistance services, workshops and e.g. the car owners who will all get benefits from it. When integrating complex and various services into a single commercial servicedriven solution, a generic infrastructure and architecture is required. Mobile clients, the core Service Por- tal and various Remote Services enable the procurement framework. They provide a market mechanism consisting of a combination of transparent service and information retrieval with concrete content and price comparisons as well as e.g. cost reduction by sharing the same infrastructure. To enable this interoperability and collaboration between independent companies, interface, communication and workflow definitions have to be specified. The Internet is used as the underlying connection means and offers high benefits regarding integration, adaptation and ubiquity. All this contributes to MYCAREVENT meeting its main objectives to strengthen competition and competitiveness among service providers in the automotive aftersales sector. $^{2}$

\section{REFERENCES}

Booth, D., Haas, H., McCabe, F., Newcomer, E., Champion, M., Ferris, C., and Orchard, D. (2004). W3C Working Group Note 11: Web Services Architecture. World Wide Web Consortium (W3C).

Bryan, M. and Wright, R. (2005). How can ontologies help repair your car. Journal of the international SGML/XML Users Group.

FIGIEFA (2002). The New Automotive Block Excemption Regulation 1400/2002/EC. FIGIEFA (International Federation of Automotive Aftermarkt Distributors).

Garg, A., Dirlenbach, H., and Quadt, A. (2004). Enabling Business Opportunities in post block exemption era MYCAREVENT. In eAdoption and the Knowledge Economy: Issues, Applications, Case Studies.

Gehlen, G., Weiss, E., Lukas, S., Rokitansky, C.-H., and Walke, B. (2006). Architecture of a vehicle communication gateway for media independent handover. In Proc. of WT2006, pages 205-209. Hamburg University of Technology.

Houben, G., den Bergh, J. V., Luyten, K., and Coninx, K. (2005a). Interactive Systems on the Road: Development of Vehicle User Interfaces for Failure Assistance. In Proc. of W-CarsCare'05, pages 84-89.

Houben, G., Fiore, F. D., Luyten, K., Reeth, F. V., and Coninx, K. (2005b). Interactive Data Units: A Framework to Support Rich Graphical Data Presentations on Heterogeneous Devices. In Proc. PSMD'05, Rome, Italy.

MYCAREVENT (2005). Deliverable 2.1: Consolidated User Survey. MYCAREVENT. http://www. mycarevent.com.

MYCAREVENT (2006). Deliverable 3.5: XML Data Structures. MYCAREVENT. http://www. mycarevent. com.

\footnotetext{
${ }^{2}$ The research at EDM (UHasselt) is partly funded by the Flemish government, EFRO, tUL and IBBT. The MYCAREVENT project FP6-IST No. 004402 is an Integrated Project sponsored by the European Commission.
} 